\title{
Reentrant disorder-disorder transitions in generalized multicomponent Widom-Rowlinson models
}

\author{
Roman Krčmár and Ladislav Šamaj \\ Institute of Physics, Slovak Academy of Sciences, \\ Dúbravská cesta 9, SK-84511 Bratislava, Slovakia
}

(Dated: August 9, 2021)

\begin{abstract}
In the lattice version of the multicomponent Widom-Rowlinson (WR) model, each site can be either empty or singly occupied by one of $M$ different particles, all species having the same fugacity $z$. The only nonzero interaction potential is a nearest-neighbor hard-core exclusion between unlike particles. For $M<M_{0}$ with some minimum $M_{0}$ dependent on the lattice structure, as $z$ increases from 0 to $\infty$ there is a direct transition from the disordered (gas) phase to a demixed (liquid) phase with one majority component at $z>z_{d}(M)$. If $M \geq M_{0}$, there is an intermediate ordered "crystal phase" (composed of two nonequivalent even and odd sublattices) for $z$ lying between $z_{c}(M)$ and $z_{d}(M)$ which is driven by entropy. We generalize the multicomponent WR model by replacing the hard-core exclusion between unlike particles by more realistic large (but finite) repulsion. The model is solved exactly on the Bethe lattice with an arbitrary coordination number. The numerical calculations, based on the corner transfer matrix renormalization group, are performed for the twodimensional square lattice. The results for $M=4$ indicate that the second-order phase transitions from the disordered gas to the demixed phase become of first order, for an arbitrarily large finite repulsion. The results for $M \geq M_{0}$ show that, as the repulsion weakens, the region of crystal phase diminishes itself. For weak enough repulsions, the direct transition between the crystal and demixed phases changes into a separate pair of crystal-gas and gas-demixed transitions; this is an example of a disorder-disorder reentrant transition via an ordered crystal phase. If the repulsion between unlike species is too weak, the crystal phase disappears from the phase diagram. It is shown that the generalized WR model belongs to the Ising universality class.
\end{abstract}

PACS numbers: 64.60.Cn, 05.50.+q, 75.10.Hk

\section{INTRODUCTION}

The nearest-neighbors lattice gas analogy of Onsager's solution of the two-dimensional Ising model enabled one to understand physical implications of the spontaneous breaking of the particle-hole symmetry on the existence and critical properties of high-density liquid and lowdensity vapor phases [1].

As concerns continuum fluids in thermal equilibrium, Widom and Rowlinson [2] introduced a simple model of identical particles (molecules) living in an infinite $\nu$ dimensional space of points $\mathbf{r} \in V \rightarrow \mathrm{R}^{\nu}$. There is a sphere of radius $R$ and volume $v_{0}$ around the center of each molecule. The potential energy $U$ associated with a given configuration $\mathbf{r}_{1}, \ldots, \mathbf{r}_{N}$ of $N$ molecules is defined by

$$
U\left(\mathbf{r}_{1}, \ldots, \mathbf{r}_{N}\right)=\epsilon\left[\frac{V\left(\mathbf{r}_{1}, \ldots, \mathbf{r}_{N}\right)}{v_{0}}-N\right],
$$

where $V\left(\mathbf{r}_{1}, \ldots, \mathbf{r}_{N}\right)$ denotes the volume covered by the corresponding $N$ (in general penetrating) spheres and $\epsilon>0$ is some energy constant. Due to the obvious inequalities $v_{0} \leq V\left(\mathbf{r}_{1}, \ldots, \mathbf{r}_{N}\right) \leq N v_{0}$, the potential energy is bounded as follows

$$
-(N-1) \epsilon \leq U\left(\mathbf{r}_{1}, \ldots, \mathbf{r}_{N}\right) \leq 0 .
$$

The lower bound ensures a correct thermodynamics, the upper bound tells us that the short-range forces among molecules are purely attractive. The model is studied within the grand canonical ensemble characterized by the dimensionless inverse temperature $\theta=\epsilon /\left(k_{\mathrm{B}} T\right)$ and the particle fugacity $z$. The corresponding particle density is given by $\rho(z, \theta)=v_{0}\langle N\rangle / V$; $z$ is normalized so as to be asymptotically equal to $\rho$ in the ideal gas limit $\rho \rightarrow 0$.

The symmetry of the Widom-Rowlinson (WR) fluid, whose spontaneous breaking is responsible for the existence of liquid and vapor phases, is hidden in the original formulation. It becomes transparent after mapping (in a thermodynamic sense) the WR model onto a WR mixture of two kinds of molecules $\sigma \in\{A, B\}$ interacting in a pairwise manner $U(\{\mathbf{r}\})=\sum_{i<j} u_{\sigma_{i} \sigma_{j}}\left(\left|\mathbf{r}_{i}-\mathbf{r}_{j}\right|\right)$, where the particles of the same species do not interact whereas the unlike species interact with a hard-core repulsion at distances smaller than $2 R$,

$$
u_{\sigma \sigma^{\prime}}(r)= \begin{cases}\infty & \text { if } \sigma \neq \sigma^{\prime} \text { and } r<2 R, \\ 0 & \text { otherwise. }\end{cases}
$$

Let us consider the case of equivalent species fugacities $z_{A}=z_{B}=z$. At very low $z$, the system behaves like the ideal gas with just one pure (mixed) phase with equivalent species densities $\rho_{A}=\rho_{B}$. At very large $z$, since unlike molecules experience an infinitely strong repulsion, the mixed phase suffers from packing effects which are substantially reduced in a demixed phase with a single majority component. Consequently, the $A-B$ symmetry is broken and the WR mixture can exist in two different homogeneous (i.e. translationally invariant) pure phases: the $A$-rich phase with $\delta \rho \equiv \rho_{A}-\rho_{B}>0$ or the $B$-rich phase with $\delta \rho<0$. The two phases become equivalent 
$(\delta \rho=0)$ at the "demixing" critical point $z_{d}$. For dimensions $\nu \geq 2$, the proof of the existence of more than one pure thermodynamic phase for sufficiently large $z$ was given by Ruelle [3] using the Peierls contour method. Ruelle's proof was generalized by Lebowitz and Lieb [4] to the case when $u_{A B}(r)(r<2 R)$ is large positive but not infinite. Integral equation theories for the pair correlation functions of the WR mixture were developed in Refs. [5, 6].

The multicomponent generalization of the WR $A-B$ mixture consists in considering molecules of $M$ different types $\sigma=1, \ldots, M$ with the same fugacity $z$. The molecules interact in a pairwise manner, the only interaction is the hard-sphere repulsion between any two particles of unlike species like in (3). It was shown [7, 8] that in dimensions $\nu \geq 2$ the WR model with any finite number of components $M$ exhibits the demixing phase transition at some $z_{d}(M)$; in a pure demixed phase, the homogeneous density of just one of the components is dominant, say $\rho_{1}>\rho_{2}=\rho_{3}=\ldots=\rho_{M}$. A hard (hyper-)cube version of the $M$-component WR model was studied in the limit of infinite dimensionality $\nu \rightarrow \infty[9]$; the calculation of thermodynamic functions within the second virial coefficient is exact in that limit. It turns out that for $M \geq 31$ the transition from the mixed phase at small values of $z$ to the demixed phase at large values of $z$ is preempted by solidification at intermediate values of $z$, $z_{c}(M)<z<z_{d}(M)$. In the corresponding crystal phase, all species are equivalent $\left(\rho_{1}=\rho_{2}=\ldots=\rho_{M}=\rho / M\right.$ with $\rho$ being the total density of molecules), but the density $\rho \equiv \rho(\mathbf{r})$ varies periodically in space, i.e. the translational symmetry is broken. The origin of this phenomenon is purely entropic: for large $M$ it pays the system to create a periodic structure of alternating dense and sparse regions, where the particles in the dense regions are less restricted by the hard-core repulsions coming from particles in the sparse regions.

In the lattice version of the multicomponent WR model [7, 8], each lattice site $i$ can be either empty $\left\{\sigma_{i}=\right.$ $0, z(0)=1\}$ or singly occupied by a particle of type $\sigma_{i}=1,2, \ldots, M$, all particles having the same fugacity $z(1)=z(2)=\ldots=z(M) \equiv z$. The potential energy of a state configuration $\left\{\sigma_{i}\right\}$ reads as $U\left(\left\{\sigma_{i}\right\}\right)=$ $\sum_{\langle i, j\rangle} u\left(\sigma_{i}, \sigma_{j}\right)$, where the interaction potential between nearest-neighbor sites $\langle i, j\rangle$ is given by

$$
u\left(\sigma_{i}, \sigma_{j}\right)= \begin{cases}\infty & \text { if } \sigma_{i} \neq \sigma_{j} \text { and } \sigma_{i} \neq 0, \sigma_{j} \neq 0, \\ 0 & \text { otherwise. }\end{cases}
$$

The number density of species $\sigma=1, \ldots, M$ at site $i$ will be denoted by $\rho_{i}(\sigma)$, the total density of particles at site $i$ by $\rho_{i}=\sum_{\sigma=1}^{M} \rho_{i}(\sigma)$.

The phase diagram of the lattice WR model is similar to that of the continuous WR model. In dimensions $\nu \geq 2$ and for any finite number of components $M$, the lattice WR model exhibits the demixing phase transition at some $z_{d}(M)$; in a pure demixed phase, the site-independent density of just one of the components is dominant, say $\rho(1)>\rho(2)=\ldots=\rho(M)$. The effect of varying exclusion diameter between different species was studied in [10]. When the number of components $M$ is equal to or larger than some minimum $M_{0}$, an entropydriven crystal phase exists for $z_{c}(M)<z<z_{d}(M)$; the transition at $z_{c}(M)$ is always of second order. In the crystal phase, the average total particle densities on the even and odd sublattices are unequal, $\rho_{e} \neq \rho_{o}$, while the average densities of the species $\sigma=1,2, \ldots, M$ are the same within a given sublattice, i.e. $\rho_{e}(\sigma)=\rho_{e} / M$ and $\rho_{o}(\sigma)=\rho_{o} / M$. The rigorous upper bound $M_{0}<27^{6}$ derived for the square lattice [8] was surprisingly large. The exact solution for the Bethe lattice of coordination $q$ gives $M_{0}=[q /(q-2)]^{2}$ which would suggest more realistic $M_{0} \sim 4$ for $q=4$, while the Monte-Carlo (MC) simulations for the square lattice imply $M_{0}=7[11]$. The extension of the hard-core exclusion to next-to-nearestneighbors leads to analogous phases whose numbers and characters depend on the specific lattice geometry [12].

The infinite hard-core potential is a mathematical simplification of real inter-particle interactions. In this paper, we generalize the multicomponent WR model by replacing the hard-core exclusion between unlike species by an arbitrary repulsion. Namely, the infinity in the interaction potential (4) between unlike species on the nearest-neighbor sites is replaced by $U \geq 0$. The corresponding interaction Boltzmann factor

$$
j=\exp (-\beta U)
$$

lies in the interval $[0,1]$. The case $j=0(U \rightarrow \infty)$ corresponds to the standard multicomponent WR model. The opposite extreme case $j=1(U=0)$ is equivalent to the non-interacting $M$-component lattice gas of singly occupied lattice sites, with the trivial grand partition function for a lattice of $N$ sites:

$$
\Xi=(1+M z)^{N} .
$$

We shall concentrate on the neighborhood of the WR point $j=0$, i.e. on large but finite repulsions, and study fundamental effects of nonzero $j$ on the phase diagram.

The model is solved exactly for the Bethe lattice with an arbitrary coordination number $q$. The numerical calculations, based on the corner transfer matrix renormalization group (CTMRG), are performed for the twodimensional square lattice. The results for $M=4$ indicate that the second-order phase transitions from the disordered gas to the demixed phase become of first order, for an arbitrarily small positive $j$. The results for $M \geq M_{0}$ show that, as the repulsion weakens, the region of crystal phase diminishes itself. For weak enough repulsions, the direct transition between the crystal and demixed phases changes into a separate pair of crystalgas and gas-demixed transitions; this is an example of a disorder-disorder reentrant transition via an ordered crystal phase. If the repulsion between unlike species is too weak, the crystal phase disappears from the phase diagram.

A reentrant fluid-solid-fluid transition was observed in previous studies of systems with soft cores and two re- 
pulsive interaction ranges, like the two-scale ramp potential [13, 14] or the square well-square shoulder model [15, 16]. The computer simulations of the purely repulsive ramp potential [17, 18] show a structural anomaly, namely the melting of the solid phase when the pressure is increased along an isotherm. This phenomenon was detected also in Stillinger's Gaussian core model [19, 20] and the antiferromagnetic Ising model with a nearestneighbor interaction and a staggered mean field [21], and later on in models with one-scale interactions [22, 23]. The structural anomaly is shared by real physical systems like water, silica or phosphorus [24, 25]. Different definitions of the structural anomaly in fluids, which can lead to very different results, was discussed in [26]. In all mentioned papers, the fluid-solid and solid-fluid transitions are discontinuous (of first order), with regions of the phase coexistence. On the other hand, the disordercrystal and crystal-disorder transitions of the generalized WR model are always continuous (of second order).

The paper is outlined as follows. In Sec. II, we present the exact solution of the model on the Bethe lattice with the coordination number $q$. For the two-dimensional square lattice, the CTMRG technique is explained in Sec. III. A. Sec. III. B brings the numerical results. Conclusions are given in Sec. IV.

\section{BETHE LATTICE COMPUTATION}

We consider the Bethe lattice of (locally equivalent) sites deep inside a tree, with coordination number $q$. The present calculation is based on the exact solution of an "inverse problem" for simply connected lattice structures [27, 28]; for details in the $j=0$ case, see Ref. 11].

Every site $i$ of the Bethe lattice is an articulation point of multiplicity $q$. Site $i$ can be either empty, $\sigma=0$, or occupied by one of the particles of $M$ different types, $\sigma=1, \ldots, M$. The corresponding set of fugacities reads as $z_{i}(0)=1$ and $z_{i}(\sigma)=z$ for $\sigma=1, \ldots, M$. For a given statistical model with two-site interactions between nearest-neighbor sites, we calculate the mean particle densities $\left\{\rho_{i}(\sigma)\right\}$, constrained by

$$
\sum_{\sigma=0}^{M} \rho_{i}(\sigma)=1
$$

for each lattice site $i$. The direct problem, find $\left\{\rho_{i}(\sigma)\right\}$ for given $\left\{z_{i}(\sigma)\right\}$, is nonlocal. The inverse problem, find $\left\{z_{i}(\sigma)\right\}$ for prescribed $\left\{\rho_{i}(\sigma)\right\}$, is local in the sense that $z_{i}(\sigma)$ depends on $\rho_{i}(\sigma)$ at the same site and on $\left\{\rho_{j}(\sigma)\right\}$ at the nearest-neighbor sites $j=1, \ldots, q$. According to [27, 28], the inverse solution for the Bethe lattice can be constructed from local inverse problems for nearestneighbor pairs of sites.

- Let $z_{i}^{\langle i, j\rangle}(\sigma)\left(z_{j}^{\langle i, j\rangle}(\sigma)\right)$ be the set of fugacities at site $i(j)$ which produce the given density profiles $\rho_{i}(\sigma)$ and $\rho_{j}(\sigma)$ at nearest-neighbor sites $\langle i, j\rangle$. The fugacities at the original Bethe lattice are then expressible as

$$
z_{i}(\sigma)=\left[\frac{\rho_{i}(0)}{\rho_{i}(\sigma)}\right]^{q-1} \prod_{j=1}^{q} z_{i}^{\langle i, j\rangle}(\sigma)
$$

- Similarly, denoting by $\Xi^{i}$ the one-site and by $\Xi^{\langle i, j\rangle}$ the two-site grand partition function, the grand potential on the whole lattice is given by

$$
\beta \Omega=-\sum_{\langle i, j\rangle} \ln \Xi^{\langle i, j\rangle}+(q-1) \sum_{i} \ln \Xi^{i} .
$$

In the two-site problems, the expected symmetry breaking of the particle densities must be reflected via an analogous symmetry breaking in the corresponding fugacities.

\section{A. Crystal phase}

In the crystal phase, there are two alternating even and odd sublattices. For the particle states $\sigma=1, \ldots, M$, we set $\rho_{i}(\sigma)=\rho_{e}$ for even sites and $\rho_{i}(\sigma)=\rho_{o}$ for odd sites. In the two-site $\langle i, j\rangle$ problem, we have $z_{i}^{\langle i, j\rangle}(0)=$ $z_{j}^{\langle i, j\rangle}(0)=1$ and $z_{i}^{\langle i, j\rangle}(\sigma)=z_{1}, z_{j}^{\langle i, j\rangle}(\sigma)=z_{2}$ for $\sigma=$ $1, \ldots, M$. The two-site grand partition function $\Xi^{\langle i, j\rangle} \equiv$ $\Xi^{\langle 1,2\rangle}$ reads as

$$
\Xi^{\langle 1,2\rangle}=1+M\left(z_{1}+z_{2}\right)+M z_{1} z_{2}+M(M-1) j z_{1} z_{2},
$$

where the first term comes from two empty sites, the second one from one empty site and the other occupied by an arbitrary particle, the third one from two particles in the same state and the fourth one from two particles in different states. The corresponding one-site particle densities are given by

$$
\begin{aligned}
& \rho_{1} \Xi^{\langle 1,2\rangle}=z_{1}+z_{1} z_{2}+(M-1) j z_{1} z_{2}, \\
& \rho_{2} \Xi^{\langle 1,2\rangle}=z_{2}+z_{1} z_{2}+(M-1) j z_{1} z_{2} .
\end{aligned}
$$

After some algebra, these equations determine $z_{1}$ and $z_{2}$ as functions of the particle densities as follows

$$
\begin{aligned}
& z_{1}=\frac{M\left(\rho_{1}+\rho_{2}\right)-1+\left(\rho_{1}-\rho_{2}\right)[1+j(M-1)]+\sqrt{D}}{2\left(1-M \rho_{1}\right)[1+j(M-1)]}, \\
& z_{2}=\frac{M\left(\rho_{1}+\rho_{2}\right)-1+\left(\rho_{2}-\rho_{1}\right)[1+j(M-1)]+\sqrt{D}}{2\left(1-M \rho_{2}\right)[1+j(M-1)]},
\end{aligned}
$$

where the plus sign of the square root of the discriminant

$$
\begin{aligned}
D= & {\left[1-(M-1)(1-j)\left(\rho_{1}+\rho_{2}\right)\right]^{2} } \\
& +4 \rho_{1} \rho_{2}(M-1)(1-j)[1+j(M-1)]
\end{aligned}
$$


is fixed by the condition $z_{1,2} \rightarrow 0$ for $\rho_{1,2} \rightarrow 0$. For $\Xi^{\langle 1,2\rangle}$, we get

$$
\begin{aligned}
\Xi^{\langle 1,2\rangle}= & \frac{M(M-1)(1-j)\left(\rho_{1}+\rho_{2}\right)}{2\left(1-M \rho_{1}\right)\left(1-M \rho_{2}\right)[1+j(M-1)]} \\
& +\frac{2[1+j(M-1)]+M(\sqrt{D}-1)}{2\left(1-M \rho_{1}\right)\left(1-M \rho_{2}\right)[1+j(M-1)]} .
\end{aligned}
$$

The original lattice fugacity in (8) does not depend on site $i$ for all particle states $\sigma=1, \ldots, M, z_{i}(\sigma)=z$. Using the density constraint (77), we obtain one equation for each of two sublattices:

$$
\begin{aligned}
& z=\left(\frac{1-M \rho_{1}}{\rho_{1}}\right)^{q-1} z_{1}^{q}, \\
& z=\left(\frac{1-M \rho_{2}}{\rho_{2}}\right)^{q-1} z_{2}^{q} .
\end{aligned}
$$

The grand-potential per site $\beta \omega \equiv \beta \Omega / N$ is determined from (9) as

$$
\beta \omega=-\frac{q}{2} \ln \Xi^{\langle 1,2\rangle}-\frac{q-1}{2} \ln \left[\left(1-M \rho_{1}\right)\left(1-M \rho_{2}\right)\right] .
$$

Introducing the new variables $s=\left(\rho_{1}+\rho_{2}\right) / 2$ and $t=\rho_{1}-\rho_{2}$, Eqs. (15a) and (15b) can be written as

$$
z=z(s, t)=z(s,-t) .
$$

They always have a trivial solution with $t=0$, which corresponds to the disordered phase of equivalent even and odd sublattices, $\rho_{1}=\rho_{2}$. A nontrivial solution $t \neq 0$ exists if $s \in\left[s_{c}^{L}, s_{c}^{U}\right]$, where the lower and upper bounds are given by the equation $\partial z(s, t) /\left.\partial t\right|_{t=0}=0$ [11] :

$$
\begin{aligned}
& s_{c}^{L}=\frac{1}{2 M}(1-\sqrt{E}), \\
& s_{c}^{U}=\frac{1}{2 M}(1+\sqrt{E})
\end{aligned}
$$

with

$$
E=1-\frac{4 M(q-1)}{(1-j) q^{2}(M-1)} .
$$

The corresponding critical fugacities read

$$
\begin{aligned}
& z_{c}^{L}=\frac{M^{q-1}}{[1+j(M-1)]^{q}} \frac{1-\sqrt{E}}{1+\sqrt{E}}\left(\frac{(q-2) / q-\sqrt{E}}{1-\sqrt{E}}\right)^{q}, \\
& z_{c}^{U}=\frac{M^{q-1}}{[1+j(M-1)]^{q}} \frac{1+\sqrt{E}}{1-\sqrt{E}}\left(\frac{(q-2) / q+\sqrt{E}}{1+\sqrt{E}}\right)^{q} .
\end{aligned}
$$

The value of the nontrivial $t$ changes continuously from 0 at $z \leq z_{c}^{L}$ to some nonzero symmetry-broken value $\pm T$ in the interval $\left(z_{c}^{L}, z_{c}^{U}\right)$ and finally goes again continuously to 0 at $z_{c}^{U}$. For $z>z_{c}^{U}$, the disordered phase with the trivial $t=0$ takes place.
The crystal phase can exist only if the discriminant $E \geq 0$. This means that, for the fixed coordination number $q$ and the Boltzmann factor $j<(q-2)^{2} / q^{2}$, the number of components $M$ must be equal or larger than some minimum $M_{0}, M \geq M_{0}$, given by

$$
M_{0}=\frac{(1-j) q^{2}}{(q-2)^{2}-j q^{2}} .
$$

Note that $M_{0} \rightarrow \infty$ just at $j=(q-2)^{2} / q^{2}$. Equivalently, for the fixed coordination number $q$ and the number of components $M>(q-2)^{2} / q^{2}$, the crystal phase exists only if the Boltzmann factor $j \leq j_{\max }$, where the maximum value $j_{\text {max }}$ is given by

$$
j_{\max }=1-\frac{4 M(q-1)}{q^{2}(M-1)} .
$$

In the limit of $M \rightarrow \infty$, we have the asymptotic expansion

$$
j_{\max }=\frac{(q-2)^{2}}{q^{2}}-\frac{4(q-1)}{q^{2}} \frac{1}{M}+O\left(\frac{1}{M^{2}}\right) .
$$

\section{B. Demixed phase}

In the demixed phase, all sites are equivalent but one of the components, say $\sigma=1$, is dominant. This means that the density of 1 -particles $\rho_{i}(1)=\rho(1)$ is larger than $\rho_{i}(\sigma)=\rho(2)$ for all remaining particle states $\sigma=2, \ldots, M$. In the two-site $\langle i, j\rangle$ problem, we set $z_{i}^{\langle i, j\rangle}(0)=z_{j}^{\langle i, j\rangle}(0)=1, z_{i}^{\langle i, j\rangle}(1)=z_{j}^{\langle i, j\rangle}(1)=z(1)$ for particles of type 1 and $z_{i}^{\langle i, j\rangle}(\sigma)=z_{j}^{\langle i, j\rangle}(\sigma)=z(2)$ for all particles of type $\sigma=2, \ldots, M$. The two-site grand partition function is given by

$$
\begin{aligned}
\Xi^{\langle 1,2\rangle}= & 1+2 z(1)+z(1)^{2}+2(M-1) z(2) \\
& +(M-1) z^{2}(2)+2(M-1) j z(1) z(2) \\
& +(M-1)(M-2) j z^{2}(2) .
\end{aligned}
$$

The corresponding particle densities are given by

$$
\begin{aligned}
& \rho(1) \Xi^{\langle 1,2\rangle}=z(1)+z^{2}(1)+(M-1) j z(1) z(2), \\
& \rho(2) \Xi^{\langle 1,2\rangle}=z(2)+z^{2}(2)+j z(1) z(2)+(M-2) j z^{2}(2) .
\end{aligned}
$$

Considering in (8) $z_{i}(\sigma)=z$ for all sites $i$ and particle states $\sigma=1,2, \ldots, M$, we get

$$
\begin{aligned}
& z=\left(\frac{1-\rho(1)-(M-1) \rho(2)}{\rho(1)}\right)^{q-1} z(1)^{q}, \\
& z=\left(\frac{1-\rho(1)-(M-1) \rho(2)}{\rho(2)}\right)^{q-1} z(2)^{q} .
\end{aligned}
$$

The grand-potential per site is obtained in the form

$$
\beta \omega=-\frac{q}{2} \ln \Xi^{\langle 1,2\rangle}-(q-1) \ln [1-\rho(1)-(M-1) \rho(2)] .
$$




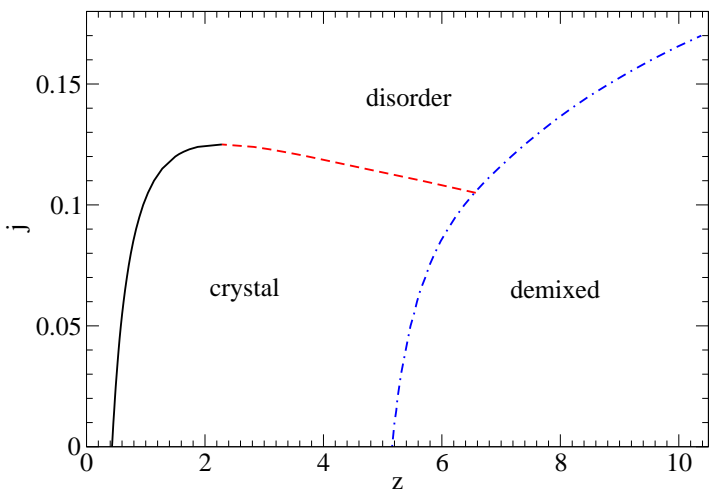

FIG. 1. (Color online) The phase diagram in the $(z, j)$ plane for the generalized WR model on the Bethe lattice with the coordination number $q=4$ and the number of components $M=7$.

The above set of nonlinear equations can be solved only numerically. The trivial disordered solution $\rho(1)=\rho(2)$ always exists. If more real solutions exist, the one with the minimal $\beta \omega$ dominates.

Let $M_{t} \leq M_{0}$ denote the "transition" number of components, such that the direct transition from the disordered to the demixed phase is of second order for $M \leq M_{t}$ and of first order for $M>M_{t}$. For the Bethe lattice of the coordination number $q$, we have the trivial value $M_{t}=2$ independent of $j$.

In Fig. 1 we present the phase diagram in the $(z, j)$ plane for our generalized WR model on the Bethe lattice with the coordination number $q=4$ and the number of components $M=7$. Note that from Eq. (21) we have $M_{0}=4$ for $j=0$ and $M_{0}=7$ for $j=1 / 8$, i.e. there is no crystal phase for $M=7$ components if $j>1 / 8$. The second-order transition line from the disorder to crystal phases, given by formula (20a), is depicted by the solid curve. The second-order transition line from the crystal to disorder phases, given by formula (20b), is depicted by the dashed curve; along this line the system exhibits the reentrant phenomenon. The transitions from the crystal or disordered phases to the demixed phase take place along the dash-dotted curve. It is seen that for small $j \lesssim 0.105$, the successive order in which the phase transitions take place is basically the same as in the standard WR model with $j=0$. For weak enough repulsions $j \in[0.105,0.125)$, the direct transition between the crystal and demixed phases changes into a separate pair of crystal-gas and gas-demixed transitions; this is an example of a disorder-disorder reentrant transition via an ordered crystal phase. If the repulsion between unlike species is too weak $j>1 / 8$, the crystal phase disappears from the phase diagram.

\section{CTMRG METHOD}

\section{A. Technique}

On the square lattice, the free energy $\mathcal{Z}$ can be decomposed into four corner transfer matrices $\mathcal{C}$, each representing the Boltzmann weight of a quadrant of the lattice system $\mathcal{Z}=\operatorname{Tr} \mathcal{C}^{4}[29]$. The fourth power of the corner transfer matrix can be then interpreted as the density matrix $\rho=\mathcal{C}^{4}$. The concept of the renormalization can be applied to this density matrix [30 32]. The CTMRG method combines the corner transfer matrix representation of the density matrix, the density matrix renormalization and an accurate approximation of the free energy for a large scale system in terms of an iterative numerical calculation [33 35]. During the process, the space of states is truncated. The dimension of the truncated space is denoted by $D$; the larger value of $D$ is used, the more accurate results are obtained.

We consider a square of lattice sites with dimension $L \times L$. There is a central row and $N$ additional rows on both sides, so $L=2 N+1$ where $N$ counts for the number of iterations of the transfer matrix. When the density matrix $\rho^{(N)}$ is obtained for a sufficiently large system, we calculate the expectation values of microscopic variables at the center of the system, which represents bulk thermodynamics deep inside the system. For example, the spontaneous particle density for our crystal phase is obtained as follows

$$
m_{c}^{(N)}=\frac{1}{\operatorname{Tr} \rho^{(N)}} \operatorname{Tr}\left\{[2 n(i, j)-1](-1)^{i+j} \rho^{(N)}\right\},
$$

where $\{i, j\}$ are the coordinates of the central point and the function $n(i, j)=0$ if the central site is empty and $n(i, j)=1$ otherwise. This expression reproduces correctly $m_{c}$ as the sublattices $A$ and $B$ difference $m_{A}-m_{B}$. As the order parameter for the demixed phase, we consider

$$
m_{d}=\left|m_{1} \mathrm{e}^{-\mathrm{i} \phi_{1}}+\cdots+m_{M} \mathrm{e}^{-\mathrm{i} \phi_{M}}\right|,
$$

where $m_{\sigma}$ is the occupation of the particle state $\sigma=$ $1,2, \ldots, M$ and the angle $\phi_{\sigma}=2 \pi(\sigma-1) / M$. The quantity $m_{d}$ vanishes in the disordered phase with $m_{1}=m_{2}=$ $\cdots=m_{M}$ and attains a positive value if one of the components is dominant. In the limit $z \rightarrow \infty, m_{d}=1$ for any value of $M \geq 2$ and $j \in[0,1]$.

In order to detect the position of the phase transitions, special quantities exhibiting singular behavior near the phase transition, like the specific heat, are usually used. Here, we use the von Neumann entropy, defined as

$$
S_{N}=-\operatorname{Tr} \rho \ln \rho .
$$

Close to a second-order critical point, the von Neumann entropy can be expressed as [36, 37]

$$
S_{N} \sim \frac{c}{6} \ln \xi,
$$




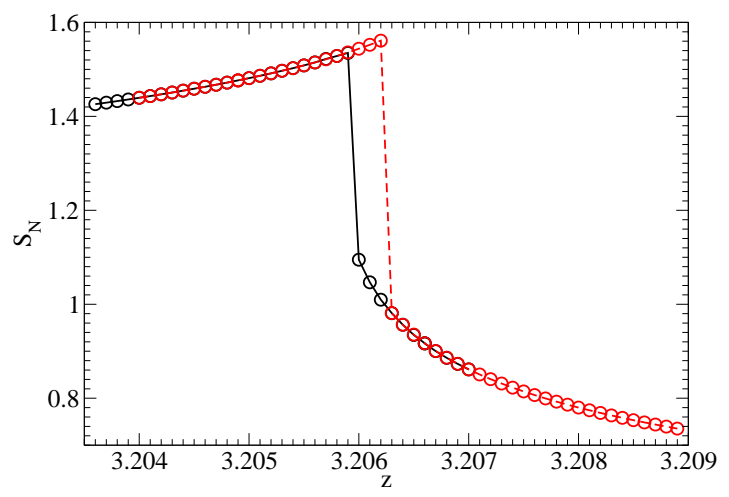

FIG. 2. (Color online) The von Neumann entropy of the disorderdemixed phase transition for the generalized WR model with $M=$ 4 components and a large finite repulsion $j=0.001$. The solid and dashed curves correspond to fixed and free boundary conditions, respectively.

where $c$ is the central charge and $\xi$ is the correlation length of the particle system. Consequently, the von Neumann entropy has a logarithmic divergence at the critical point. At a first-order transition point, it exhibits a discontinuity.

As concerns critical exponents, we shall calculate the plot of the spontaneous density around the critical point to obtain the exponent $\beta$. Moreover, in two dimensions and at the critical point, we shall study the finite-size scaling of the particle density

$$
m(L) \propto L^{-\eta / 2}, \quad \text { large sample size } L,
$$

to deduce the exponent $\eta$ 34]. Using, e.g., the numerical logarithmic derivative

$$
\eta_{e f f}(L)=-2 \frac{\ln [m(L+2)]-\ln [m(L)]}{\ln (L+2)-\ln (L)},
$$

we can extract the critical exponent $\eta$ as the limit $\eta=$ $\lim _{L \rightarrow \infty} \eta_{\text {eff }}(L)$.

\section{B. Numerical results}

The MC simulations for $j=0$ in Ref. [11] indicate that at the direct disorder-demixed phase transition $z_{d}$, there is no jump in the density for $M \leq 4$ (so the transition is of second-order) while there is a jump in density for $M \geq 5$. In other words, $M_{t}=4$ for the standard WR model on the square lattice. For $M=4$ particle components, an arbitrarily large (but finite) repulsion between different species causes that the disorder-demixed transition becomes of first order. This fact is documented in Fig. 2 on the plot of the von Neumann entropy versus the fugacity for the generalized WR model with $M=4$ components and a large finite repulsion $j=0.001$. The solid (black) curve connects data evaluated with boundary conditions fixed at the particle state dominant in the demixed phase while the dashed (red) curve connects

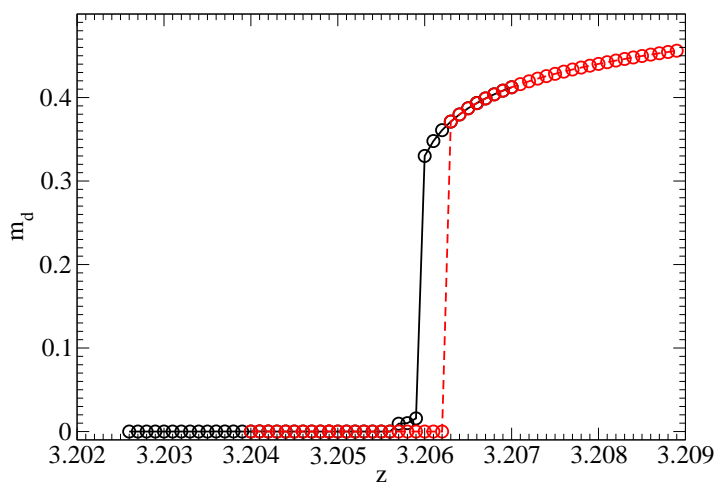

FIG. 3. (Color online) The spontaneous particle density of the disorder-demixed transition for $M=4$ and $j=0.001$.

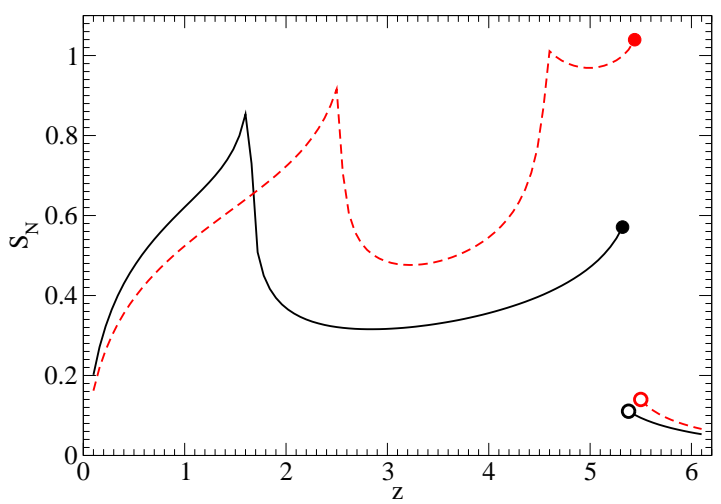

FIG. 4. (Color online) The von Neumann entropy $S_{N}$ versus the fugacity $z$ for the WR model with $M=7$ particle components. The solid curve corresponds to an infinite $j=0$ repulsion between unlike species, the dashed curve corresponds to a finite repulsion $j=0.018$. Dimension of truncated space of states is $D=50$.

data for free boundary conditions. We see that the two curves calculated with distinct boundary conditions produce a hysteresis inside which the disorder and demixed phase coexists, as is usual in the case first-order phase transitions [38]. The corresponding plot of the order parameter $m_{d}$ (29) is presented in Fig. 3. For $M=3$ components, the second-order phase transition takes place also for small $j>0$.

The MC simulations for $j=0$ in Ref. [11] indicate that the standard WR model on the square lattice exhibits the crystal phase at and beyond $M_{0}=7$ particle components. In Fig. 4 we plot by solid curve the von Neumann entropy $S_{N}$ versus the fugacity $z$ for that standard $j=0 \mathrm{WR}$ model with $M=7$ components. There are two singularities. The logarithmic one on the left indicates the continuous (second-order) disordercrystal phase transition, the right one indicates the discontinuous (first-order) crystal-demixed phase transition. For the generalized WR model with $j=0.018$ (dashed curve), there are three singularities. The one in the middle corresponds to the reentrant transition from the crystal to gas phase. The corresponding plots of the order parameters $m_{c}(28)$ and $m_{d}(29)$ are presented in Fig. 5 . 


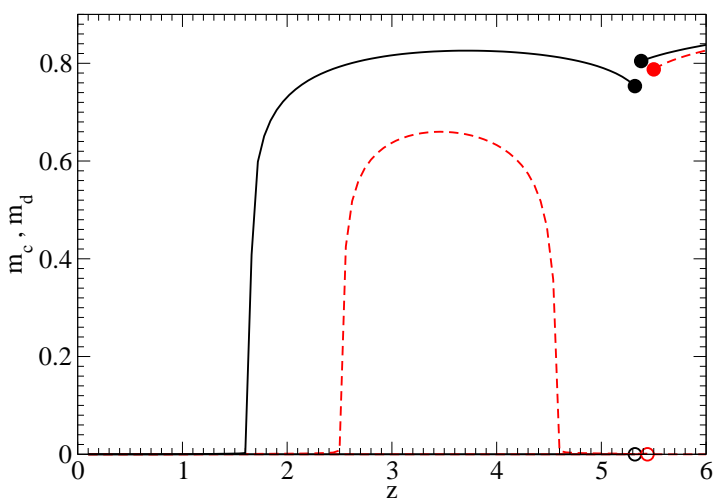

FIG. 5. (Color online) The crystal order parameter $m_{c}$ (left fragment) and the demixed order parameter $m_{d}$ (right fragment) for the Widom-Rowlinson model with $M=7$ components. The solid and dashed curves correspond to $j=0$ and $j=0.018$, respectively. $D=50$.

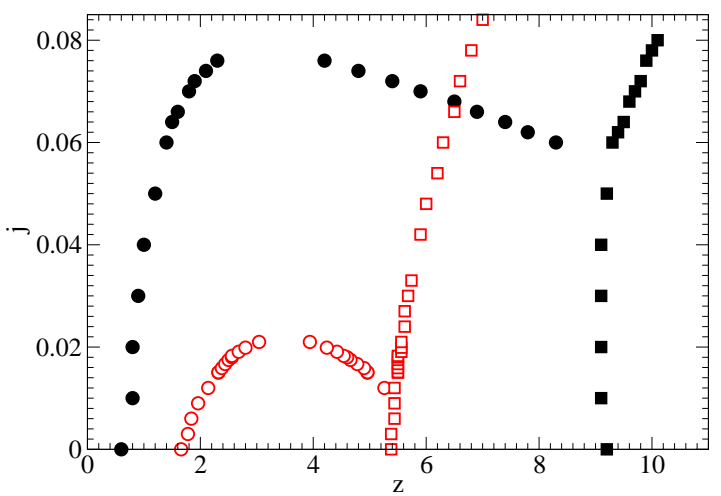

FIG. 6. (Color online) The critical lines in the $(z, j)$ plane for the generalized WR model with $M=7$ (open symbols) and $M=10$ (full symbols) components. $D=30$.

In Fig. 6, the phase diagrams in the $(z, j)$ plane are presented for the generalized WR model with $M=7$ (open symbols) and $M=10$ (full symbols) components. The disorder-crystal and crystal-disorder phase transitions are represented by circles, the crystal-demixed and disorder-demixed phase transitions are denoted by squares. The phase diagrams resemble the one for the Bethe lattice in Fig. 1

For the generalized $M$-component WR model on the Bethe lattice with the coordination number $q$ in Sec. II, we have defined the maximal repulsion Boltzmann factor $j_{\max }$, for which the crystal phase exists. For $q=4$, the exact formula (22) implies

$$
j_{\max }=\frac{1}{4}-\frac{3}{4} \frac{1}{M-1} \underset{M \rightarrow \infty}{\sim} \frac{1}{4}-\frac{3}{4} \frac{1}{M} .
$$

The corresponding dependence of $j_{\max }$ on the number of components $M$ for the Bethe lattice with $q=4$ is represented in Fig. 7 by dashed curve. The solid curve is the (inverse) polynomial fit of the numerical CTMRG data for the square lattice (solid circles). In the asymptotic

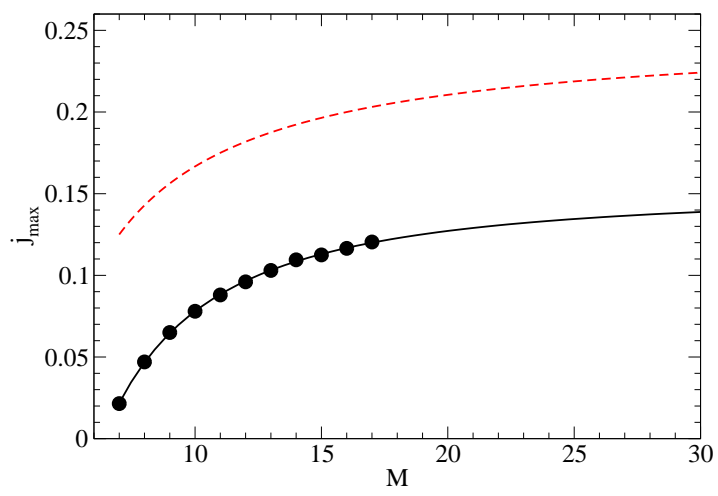

FIG. 7. (Color online) The maximal repulsion Boltzmann factor $j$ for which the crystal phase exists in the generalized WR model on the square lattice with $M$ components. The dashed curve corresponds to the exact Bethe result (22) for the coordination number $q=4$. The solid curve is the (inverse) polynomial fit of the numerical CTMRG data (solid circles) which converges to $j_{\max }=0.15$ as $M \rightarrow \infty . D=30$.

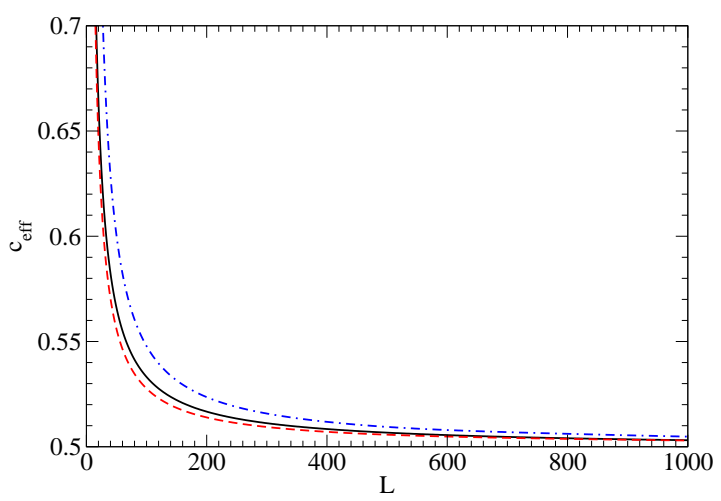

FIG. 8. (Color online) The WR model on the square lattice with $M=7$ components. The dependence of $c_{e f f}$ (36) on the system size $L$. The solid curve stands for the standard $j=0 \mathrm{WR}$ model at $z_{c}=1.66196$, the dashed curve corresponds to $\left(j=0.018, z_{c}^{L}=\right.$ $2.58545)$ and finally the dash-dotted curve corresponds to $(j=$ $\left.0.018, z_{c}^{U}=4.47455\right)$. As $L \rightarrow \infty$, all three curves converge to the central charge $c=1 / 2$ of the Ising universality class. $D=300$.

limit $M \rightarrow \infty$, the fit implies that

$$
j_{\max } \underset{M \rightarrow \infty}{\sim} 0.15-\frac{2.9}{M^{1.6}} .
$$

At the critical point, the correlation length of the particle system $\xi$ diverges in the bulk and $\xi \propto L$ for a finite system of characteristic length $L$. According to Eq. (31), defining

$$
c_{e f f}(L)=6 \frac{\partial S_{N}}{\partial(\ln L)},
$$

the central charge $c$ of the critical model is obtained as the limit $c=\lim _{L \rightarrow \infty} c_{e f f}(L)$. In what follows, we shall restrict ourselves to the WR model on the square lattice with $M=7$ components. For $j=0$, there is the only critical point $z_{c}=1.66196$ at which the disorder-crystal phase transition takes place. For $j=0.018$, there is the 


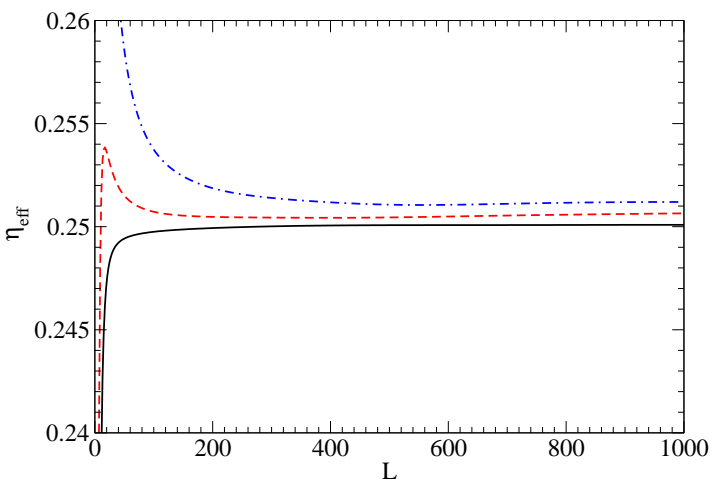

FIG. 9. (Color online) The WR model on the square lattice with $M=7$ components. The dependence of $\eta_{\text {eff }}$ (33) on the system size $L$. The solid, dashed and dash-dotted curves stand for the $(j=$ $\left.0, z_{c}=1.66196\right),\left(j=0.018, z_{c}^{L}=2.58545\right)$ and $\left(j=0.018, z_{c}^{U}=\right.$ $4.47455)$ critical points, respectively. All three curves converge to the Ising value $\eta=1 / 4$. $D=300$.

disorder-crystal phase transition at $z_{c}^{L}=2.58545$ and the reentrant crystal-disorder phase transition at $z_{c}^{U}=$ 4.47455. In Fig. 8, we plot the dependence of $c_{e f f}$ on the system size $L$ for the above three critical points: the solid curve stands for $\left(j=0, z_{c}=1.66196\right)$, the dashed curve corresponds to $\left(j=0.018, z_{c}^{L}=2.58545\right)$ and the dashdotted curve corresponds to $\left(j=0.018, z_{c}^{U}=4.47455\right)$. It is seen that as $L$ goes to infinity, all three curves converge to the value $c=1 / 2$ which is the central charge of the Ising universality class. Note that due to relatively large values of $L$ we have to increase the dimension of the truncated space to $D=300$.

To confirm the Ising universality class, we have determined the critical index $\eta$ by studying the convergence of $\eta_{e f f}$, given by formula (33), as $L \rightarrow \infty$. The results for the WR model with $M=7$ components at the three considered critical points are presented in Fig. 9, As before, the solid, dashed and dash-dotted curves stands for the $\left(j=0, z_{c}=1.66196\right),\left(j=0.018, z_{c}^{L}=2.58545\right)$ and $\left(j=0.018, z_{c}^{U}=4.47455\right)$ critical points, respectively. As $L \rightarrow \infty$, all three curves converge close to the $2 \mathrm{D}$ Ising value $\eta=1 / 4$.

As concerns the crystal order parameter $m_{c}$, we anticipate the Ising behavior

$$
m_{c} \propto\left|T-T_{c}\right|^{\beta}, \quad \beta=\frac{1}{8},
$$

close to the critical temperature $T_{c}$. Since by definition the fugacity $z=\exp \left(\mu / k_{\mathrm{B}} T\right)$ with $\mu$ being the chemical potential, it holds that $T \propto 1 / \ln (z)$. We can rewrite the relation (37) as follows

$$
m_{c}^{8} \propto\left|1 / \ln z-1 / \ln z_{c}\right| .
$$

For the generalized WR model with $M=7$ components and $j=0.018$, the plot of $m_{c}^{8}$ as the function of $1 / \ln z-$ $1 / \ln z_{c}$ is drawn in Fig. 10, the left data set corresponds to the critical point $z_{c}^{U}=4.47455$ and the right data set corresponds to the critical point $z_{c}^{L}=2.58545$. We

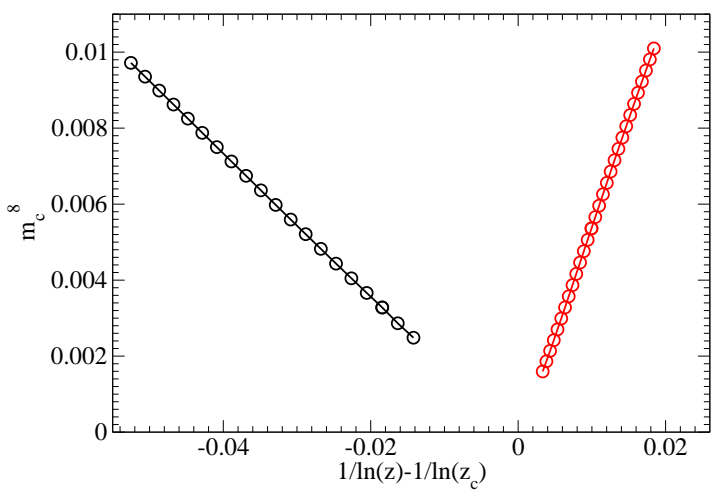

FIG. 10. (Color online) The generalized WR model with $M=7$ components and $j=0.018$. The plot of $m_{c}^{8}$ as the function of $1 / \ln z-1 / \ln z_{c}$ at the critical points $z_{c}^{U}=4.47455$ (left data) and $z_{c}^{L}=2.58545$ (right data). The linear dependence confirms the Ising critical exponent $\beta=1 / 8 . D=70$.

see that in both cases the plot is linear which confirms the Ising-like behavior (37) with the critical index $\beta=$ $1 / 8$. The knowledge of two critical exponents $\eta$ and $\beta$ determines all remaining critical indices via the scaling relations [29, 39, 40].

\section{CONCLUSION}

The lattice version of the multicomponent WR model acquired a great deal of interest because it exhibits, besides the usual demixed phase transition, also the crystal phase driven by entropy. The initial extremely large rigorous estimates of the minimum (number of components to have the crystal) $M_{0}$ were later replaced by much smaller values, 4 for the Bethe lattice with coordination $q=4$ and 7 for the 2D square lattice [11], making the WR model of practical interest.

In the original WR model, the nearest-neighbor interaction of unlike species corresponds to an infinite hardcore potential, with the corresponding Boltzmann factor $j=0$. In real physical systems with a a finite repulsion potential, $j$ is always positive. In this paper, we generalized the multicomponent WR model to an arbitrary repulsion $j \in[0,1]$. The model was studied in the region close to $j=0$, namely by using its exact solution on the Bethe lattice (with the coordination number $q=4$ ) and the numerical CTMRG technique for the square lattice. The consideration of nonzero $j$ has two fundamental effects on the phase diagram.

For $M<M_{0}$, there is a direct transition between the disordered and demixed phases. In the original WR model with $j=0$, the transition is of second order for $M=2,3,4$. It was shown here that for $M=4$ the consideration of a very small value of $j=0.001$ changes this transition to the first-order one, see Figs. 2 and 3, For $M=3$, the second order of the disorder-demixed transition remains unchanged for small $j$.

The second fundamental effect concerns the crystal 
phase. In the original WR model with $j=0$, if $M \geq M_{0}$ there are two phase transitions as the fugacity $z$ goes from 0 to $\infty$ : the disorder-crystal one at $z_{c}$ and the crystal-demixed one at $z_{d}$. In the generalized WR model with $M \geq M_{0}$, there exists certain interval of $j$-values in which the system undergoes three phase transitions: the disorder-crystal one at $z_{c}^{L}$, the crystal-disorder one at $z_{c}^{U}$ and finally the disorder-demixed one at $z_{d}$. This reentrant disorder-disorder phenomenon is interesting not only from an academic point of view. If the repulsion between unlike species is too weak, the crystal phase disappears from the phase diagram. For all studied critical points, the generalized WR model belongs to the Ising universality class with the central charge $c=1 / 2$ and the critical indices $\beta=1 / 8$ and $\eta=1 / 4$.

\section{ACKNOWLEDGMENTS}

The support received from the grant QIMABOS APVV-0808-12 and the VEGA grants Nos. 2/0130/15 and $2 / 0015 / 15$ is acknowledged.
[1] T. D. Lee and C. N. Yang, Phys. Rev. 87, 410 (1952).

[2] B. Widom and J. S. Rowlinson, J. Chem. Phys. 52, 1670 (1970).

[3] D. Ruelle, Phys. Rev. Lett. 27, 1040 (1971).

[4] J. L. Lebowitz and E. H. Lieb, Phys. Lett. A 39, 98 (1972).

[5] A. Yethiraj and G. Stell, J. Stat. Phys. 100, 39 (2000).

[6] J. M. Brader and R. L. C. Vink, J. Phys.: Condens. Matter 19, 036101 (2007).

[7] J. L. Lebowitz and G. Gallavotti, J. Math. Phys. 12, 1129 (1971).

[8] L. K. Runnels and J. L. Lebowitz, J. Math. Phys. 15, 1712 (1974).

[9] R. P. Sear, J. Chem. Phys. 104, 9948 (1996).

[10] A. Mazel, Y. Suhov, I. Stuhl, and S. Zohren, J. Stat. Mech. P08010 (2014).

[11] J. L. Lebowitz, A. Mazel, P. Nielaba, and L. Šamaj, Phys. Rev. E 52, 5985 (1995).

[12] H.-O. Georgii and V. Zagrebnov, J. Stat. Phys. 102, 35 (2001).

[13] P. C. Hemmer and G. Stell, Phys. Rev. Lett. 24, 1284 (1970).

[14] E. A. Jagla, J. Chem. Phys. 111, 8980 (1999).

[15] P. Kumar, S. V. Buldyrev, F. Sciortino, E. Zaccarelli, and H. E. Stanley, Phys. Rev. E 72, 021501 (2005).

[16] E. Lomba, N. G. Almarza, C. Martin, and C. McBride, J. Chem. Phys. 126, 244510 (2007).

[17] G. Franzese, G. Malescio, A. Skibinsky, S. V. Buldyrev, and H. E. Stanley, Phys. Rev. E 66, 051206 (2002).

[18] A. Skibinsky, S. V. Buldyrev, G. Franzese, G. Malescio, and H. E. Stanley, Phys. Rev. E 69, 061206 (2004).

[19] F. Stillinger, J. Chem. Phys. 65, 3968 (1976).

[20] A. Lang, C. N. Likos, M. Watzlawek, and H. Löwen, J. Phys.: Condens. Matter 12, 5087 (2000).

[21] J. S. Høye and E. Lomba, J. Chem. Phys. 129, 024501 (2008).

[22] F. Saija, S. Prestipino, and G. Malescio, Phys. Rev. E
80, 031502 (2009).

[23] S. Prestipino, F. Saija, and G. Malescio, J. Chem. Phys. 133, 144504 (2010).

[24] V. V. Brazhkin, S. V. Buldyrev, V. N. Ryzhov, and H. E. Stanley, New kinds of phase transitions: Transformations in disordered substances, in Proceedings of the NATO Advanced Research Workshop, Volga River (Kluwer, Dordrecht, 2002).

[25] M. S. Shell, P. G. Debenedetti, and A. Z. Panagiotopoulos, Phys. Rev. E 66, 011202 (2002).

[26] Yu. D. Fomin, V. N. Ryzhov, B. A. Klumov, and E. N. Tsiok, J. Chem. Phys. 141, 034508 (2014).

[27] L. Šamaj, J. Phys. France 50, 273 (1989).

[28] J. K. Percus and L. Šamaj, J. Stat. Phys. 77, 421 (1994).

[29] R. J. Baxter, Exactly solved models in statistical mechanics (Academic Press, London, 1982).

[30] S. R. White, Phys. Rev. Lett. 69, 2863 (1992).

[31] S. R. White, Phys. Rev. B 48, 10345 (1993).

[32] U. Schollwöck, Rev. Mod. Phys. 77, 259 (2005).

[33] T. Nishino and K. Okunishi, J. Phys. Soc. Jpn. 65, 891 (1996).

[34] T. Nishino, K. Okunishi, and M. Kikuchi, Phys. Lett. A 213, 69 (1996).

[35] T. Nishino and K. Okunishi, J. Phys. Soc. Jpn. 66, 3040 (1997).

[36] P. Calabrese and J. Cardy, J. Stat. Mach.: Theor Exp., P06002 (2004).

[37] E. Ercolessi, S. Evangelisti, and F. Ravanini, Phys. Lett. A 374, 2101 (2010).

[38] H. Graf and H. Löwen, Phys. Rev. E 57, 5744 (1998).

[39] S.-K. Ma, Modern Theory of Critical Phenomena (Westview Press, New York, 1976).

[40] L. Šamaj and Z. Bajnok, Introduction to the Statistical Physics of Integrable Many-body Systems (Cambridge Univ. Press, Cambridge, 2013). 